\title{
Estimulação cardíaca artificial e suas implicações na enfermagem
}

\section{Cardiac pacing in nursing}

\author{
Wilian Helber Mota ${ }^{1}$, Kelmi Cristina Saracini ${ }^{2}$, Leo Christyan Alves de Lima ${ }^{2}$, Ellen Daiane Biavatti de Oliveira Algeri ${ }^{3}$, Laurindo \\ Pereira de Souza ${ }^{4}$
}

1. Enfermeiro Especialista em Didática do Ensino Superior, Docente da Liga Acadêmica de Enfermagem em Cardiologia (LAEC) da Faculdade de Ciências Biomédicas de Cacoal (FACIMED), Cacoal, RO, Brasil. 2. Bacharel em Enfermagem pela Faculdade de Ciências Biomédicas de Cacoal (FACIMED), Cacoal, R0, Brasil. 3. Enfermeira do Hospital de Urgência e Emergência de Rondônia (HEURO), Cacoal, RO, Brasil. 4. Coordenador de Enfermagem do Centro de tratamento Intensiva Adulto (CTI) do Hospital Regional de Cacoal (HRC), Cacoal, RO, Brasil.

\section{Resumo}

Introdução: 0 coração é dotado de um sistema especial de condução de impulsos elétricos. Quando este sistema é lesado, pode-se indicar o implante de um dispositivo cardíaco artificial que propicie uma atividade elétrica mais fisiológica possível. É imprescindível a presença de enfermeiro na assistência de paciente crítico ou potencialmente crítico, sendo assim indispensável à atuação deste profissional nos cuidados ao paciente submetido ao implante de marcapasso cardíaco, pois este exige maior complexidade técnica e conhecimento científico. Objetivo: 0 objetivo desta pesquisa foi apresentar uma revisão bibliográfica sobre a estimulação artificial cárdica e suas implicações para a enfermagem. Método: Trata-se de estudo de revisão por meio de avaliação retrospectiva utilizando-se de pesquisa bibliográfica exploratória, das produções acerca de implante de marcapasso e suas implicações para a enfermagem, no período de 2005 a 2015. Resultados: Ao analisar o cenário epidemiológico atual, inferiu-se que o tipo de marcapasso mais implantado nas unidades da federação corresponde ao de câmara dupla transvenoso $62 \%$ do total de procedimentos sendo este o que apresenta a menor taxa de mortalidade. Com base nesses dados, pode-se pensar as condições clínicas do paciente pré-trans e pós-implante e expor os diagnósticos e intervenções de enfermagem mais próximos das necessidades do cliente. Conclusão: 0 enfermeiro tem total responsabilidade em procedimentos de emergência, portanto deve estar capacitado para assumir tais procedimentos, compreender a patologia de base e ter conhecimento técnico e científico quanto ao procedimento de implante de dispositivos cardíacos, suas indicações, complicações e necessidades do paciente fator fundamental para o exercício pleno da enfermagem.

Palavras-chave: Cuidados Críticos. Emergência. Marcapasso. Enfermagem.

\begin{abstract}
Introduction: The heart is equipped with a special system of conduction of electrical impulses. When this system is injured, it is possible to indicate the implantation of an artificial cardiac device that provides a more physiological electrical activity. It is essential the presence of nurses in the care of a critical or potentially critical patient, and it is therefore indispensable to perform this task in the care of the patient submitted to the implantation of a cardiac pacemaker, since this requires greater technical complexity and scientific knowledge. Objective: The objective of this research was to present a literature review on artificial cardiac stimulation and its implications for nursing. Method: This is a review study through a retrospective evaluation using exploratory bibliographic research, the productions about pacemaker implantation and its implications for nursing, from 2005 to 2015 . Results: When analyzing the current epidemiological scenario, it was observed that the type of pacemaker most implanted in the federation units correspond to that of the double transvenous chamber $62 \%$ of the total procedures, which is the one with the lowest mortality rate. Based on these data, one can think about the clinical conditions of the pre-trans and post-implant patients and expose the nursing diagnoses and interventions that are closer to the client's needs. Conclusion: The nurse has full responsibility for emergency procedures, so he must be able to take such procedures, understand the basic pathology and have technical and scientific knowledge regarding the procedure of implantation of cardiac devices, its indications, complications and patient needs so that the full exercise of nursing may take place.
\end{abstract}

Key words: Critical Care. Emergency. Pacemaker. Nursing.

\section{INTRODUÇÃO}

O coração é dotado de sistema especial para gerar impulsos elétricos rítmicos [...] e conduzir esses impulsos rapidamente por todo o coração. O impulso normalmente se origina no nodo sinoatrial, considerado o marcapasso natural do coração; ele controla os batimentos cardíacos uma vez que sua frequência de descarga é mais alta que qualquer outra porção do coração. ${ }^{1}$

Quando o processo normal de condução do coração é lesado, pode-se ter indicação quanto ao uso de estimulação cardíaca artificial. Algumas premissas devem ser lembradas quando se fala em indicações para a estimulação cardíaca artificial; a) o marcapasso definitivo será indicado sempre que existir bradicardia sintomática e irreversível ou risco de bradicardia grave relacionada a causas não removíveis); b) quando a causa é transitória ou removível, deve-se tentar tratamento farmacológico ou marcapasso temporário. ${ }^{2}$ Os Marcapassos

Correspondência: Wilian Helber Mota. Curso de Enfermagem da Faculdade de Ciências Biomédicas de Cacoal (FACIMED), Cacoal, RO, Brasil. E-mail: willyan_he@hotmail.com

Conflito de interesse: Não há conflito de interesse por parte de qualquer um dos autores.

Recebido em: 28 Dez 2016; Revisado em: 28 Mar 2017;11 Abr 2017; 8 Jun 2017; 16 Out 2017; Aceito em: 20 out 2017 
Cardíacos Artificiais (MP) são dispositivos eletrônicos de estimulação multiprogramável, têm como função proporcionar a atividade elétrica cardíaca mais fisiológica possível, sendo capazes de substituir impulsos elétricos e/ou ritmos ectópicos ${ }^{3-4}$.

O marcapasso é um dispositivo que libera estímulos elétricos para o músculo cardíaco quando este apresenta algum problema de condução. Tem por funções emitir um pulso elétrico que inicie o batimento cardíaco, manter o ritmo cardíaco regular com periodicidade compatível com a vida, detectar batimento cardíaco espontâneo e inibir a emissão do pulso elétrico ${ }^{4}$.

A estimulação cardíaca elétrica artificial, modernamente, deixou de ser apenas uma forma de salvar a vida de portadores de bloqueios atrioventriculares, passando a ser um modo de corrigir os distúrbios do ritmo cardíaco e do sincronismo atrioventricular. A preocupação foi além de prolongar a vida, mas também permitir que os pacientes atinjam uma qualidade de vida compatível com a média da população ${ }^{3}$.

O uso de marcapasso cardíaco definitivo é justificado quando o paciente portador de alteração significativa na propagação dos estímulos dos batimentos cardíacos necessita de um estímulo elétrico permanente para que haja a manutenção da contração cardíaca numa frequência ideal de batimentos por minuto ${ }^{6}$.

Estudo retrospectivo de 12 anos de implante de marcapasso no Brasil, realizado por meio do registro de marcapassos, Desfibriladorese Ressincronizadores Cardíacos (RBM) da base de dados do Departamento de Estimulação Cardíaca Artificial (DECA), revelou que a média de idade da população submetida ao procedimento foi de 63,7 anos com (DP $\pm 15,6)$, alcançando o patamar de 68,1 (DP \pm 15$) ;$ as indicações clínicas mais comuns foram a síncope, com $37,0 \%$ dos casos, seguida de tonturas $(16,8 \%)$ e pré-síncope $(13,0 \%)$, mostrando que os sintomas de baixo fluxo cerebral foram os principais motivos de implante. Insuficiência cardíaca-congestiva e bradicardia representaram cerca de $7,7 \%$ dos implantes cada uma, sendo a quarta e quinta causas do uso de marcapasso, respectivamente ${ }^{7,8}$.

Existem indicações consideradas clássicas, como a doença do nó sinusal e o bloqueio atrioventricular. Quando esses tipos de anormalidades são detectados, pode-se indicar o implante de marcapasso cardíaco artificial, para que se obtenha atividade elétrica cardíaca a mais fisiológica possível ${ }^{9}$.

No processo de composição das equipes de enfermagem que irão prestar cuidados a clientelas de alta complexidade é preciso considerar aspectos que dizem respeito à trajetória profissional, experiências prévias, qualificação específica. Tal composição requer olhar diferenciado sobre o preparo técnicocientífico dos enfermeiros, a fim de que a assistência prestada responda pelo atendimento das necessidades do cliente em todas as dimensões ${ }^{10}$.
Segundo Martins et al. (2006) $)^{11}$ o perfil do enfermeiro requer:

O reconhecimento de que toda pessoa tem direito à adequada assistência de enfermagem, que o atendimento de enfermagem ao ser humano deve ser considerado em sua totalidade e em constante interação com o meio ambiente, que o enfermeiro atua em diversos campos de ação, exercendo atividades de assistência, administração, ensino, pesquisa e integração, nos níveis primário, secundário e terciário e que a constante evolução das ciências da saúde exige do enfermeiro permanente atualização e, muitas vezes, especialização, que deve ser adquirida após a formação básica.

Em face da temática, o objetivo desta pesquisa foi apresentar uma revisão bibliográfica sobre a estimulação artificial cardíaca e suas implicações para a enfermagem.

\section{METODOLOGIA}

Trata-se de estudo de revisão por meio de avaliação restrospectiva utilizando-se de pesquisa bibliográfica exploratória, de produções acerca de implante de marcapasso e suas implicações para a enfermagem, no período de 2005 a 2015. Forma utilizados livros físicos e/ou digitais consagrados em cardiologia, e artigos das bases de dados: SCIELO, LILACs e RELAMPA escritos em português, inglês e espanhol; e sites dos principais órgãos ou sociedades na área da Cardiologia.

A pesquisa bibliográfica tem como objetivo procurar explicar um problema a partir de referências teóricas publicadas em documentos, podendo ser desenvolvida a partir de material já elaborado, constituído principalmente de livros, artigos científicos, dissertações de mestrado e teses de doutorado ${ }^{12}$.

A pesquisa exploratória não requer a elaboração de hipóteses a serem testadas no trabalho, restringindo-se a definir objetivos e buscar mais informações sobre determinado assunto de estudo. Tais estudos têm por objetivo familiarizar-se com o fenômeno ou obter uma nova percepção dele e descobrir novas ideias ${ }^{13}$.

Após análise de uma série histórica com recorte temporal de 2005 a 2015, foram encontrados 46 artigos publicados em periódicos; destes, foram selecionados 21 a partir dos critérios de inclusão, artigos publicados dentro do período definido para o estudo, que abordassem os temas critérios de implante do marcapasso, marcapasso cardíaco, marcapasso atrioventricular, cuidados e assistência de enfermagem. Foram excluídos os artigos que tratavam de marcapasso cardíaco em faixa etária fora do padrão epidemiológico mais comum para este tipo de procedimento (bebês, crianças, jovens, grávidas e adultos jovens), implante de marcapasso em animais, bem como os que fugiam da proposta do tema. 


\section{FLUXOGRAMA METODOLÓGICO}

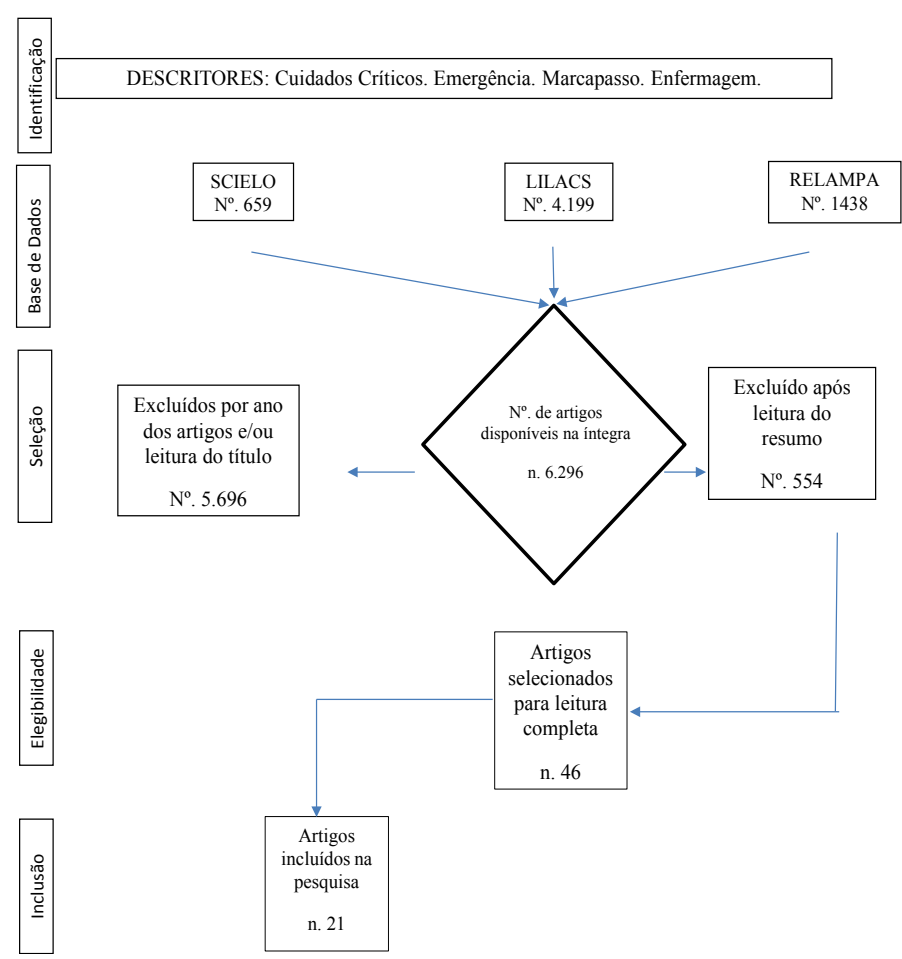

Fonte: Adaptado: Santos, JWN. Pires, DGH. Núñez, MAG. $2017^{14}$

\section{RESULTADOS E DISCUSSÃO}

\section{Conceito e Evolução Histórica}

O sistema especializado de estimulação e condução do coração consiste no nó sinoatrial (SA), no qual o impulso rítmico normal é gerado; nas vias internodais, entre os átrios e os ventrículos; no nó atrioventricular (AV) e no feixe de His, que conduzem o impulso dos átrios aos ventrículos; e nas fibras de Purkinje, que conduzem os impulsos a todas as partes do ventrículo ${ }^{15}$.

O marcapasso é um dispositivo que libera estímulos elétricos para o músculo cardíaco quando este apresenta algum problema de condução. Tem por funções emitir um pulso elétrico que inicie o batimento cardíaco, manter o ritmo cardíaco regular com periodicidade compatível com a vida, detectar batimento cardíaco espontâneo e inibir a emissão do pulso elétrico ${ }^{5}$.

Em 1883, Walter Holbrook Gaskell denominou "bloqueio cardíaco" a dissociação entre o ritmo dos átrios e o ritmo dos ventrículos decorrente da destruição da região do nóatrioventricular. Após vários estudos desenvolvidos para a tentativa de reversão de quadros clínicos de bloqueio cardíaco, finalmente, em 1930, Albert Hyman ressaltou a utilidade da estimulação cardíaca artificial quando cessada a atividade espontânea, concebendo, então, um marcapasso com gerador elétrico manual, o qual gerou a publicação de trabalhos de extrema relevância acerca do tratamento elétrico para as hipóteses de parada cardíaca. Concretizando as bases da estimulação cardíaca moderna, em 1953 ocorreu o primeiro implante de marcapasso cardíaco com fonte interna de energia, operação que foi executada por AkeSenning; e, em 1959,
Seymour Furman evidenciou a viabilidade de se proceder à estimulação endocárdica pela via transvenosa. Em meados da década de 1960, os doutores Décio Silvestre Kormann e Adib Domingos Jatene iniciaram a prática da estimulação cardíaca artificial no Brasil, com a criação de marca-passos nacionais e novas técnicas de implante. Atualmente, nos tratamentos que exigem a implantação do estimulador cardíaco ora tratado, pode-se utilizar marca-passos temporários ou definitivos ${ }^{16}$.

\section{Tipos de Marca-passos}

Os marca-passos podem ser unipolares ou bipolares. Em relação aos bipolares, o cabo-eletrodo conduz a corrente elétrica produzida no gerador de pulso até o músculo cardíaco. Para que isso ocorra, deve existir um polo positivo (ânodo) e um negativo (cátodo), por onde passa a corrente elétrica. Já nos marca-passos unipolares, o polo negativo é a extremidade distal do cabo-eletrodo, em contato direto com o coração ${ }^{17}$.

Os marca-passos podem ser de três tipos e são usados para regular uma única ou várias câmaras do coração. Um marca-passo de câmara única envolve a colocação de um único estimulador no átrio ou no ventrículo que, em seguida, pode enviar o ritmo para o átrio ou o ventrículo. Em contrapartida, o marca-passo de câmara dupla é constituído por dois estimuladores inseridos no coração. Um cabo-eletrodo pode ser inserido na aurícula direita e fixado nessa posição, regulando o átrio. Outro tipo de eletrodo pode ser inserido no ventrículo direito e fixado nessa posição. Quando ativados e em funcionamento, esses eletrodos conferem um ritmo ao átrio e ao ventrículo, sequencialmente, simulando a ação do complexo estimulante do coração normal. Este tipo de regulação é o mais comumente usado atualmente ${ }^{18}$.

A estimulação multissítio ventricular é obtida pela combinação de eletrodo posicionado por via transvenosa no ventrículo direito com outro colocado na via de saída desta câmara (forma dita bifocal, eventualmente utilizada), com eletrodo epimocárdico posicionado em ventrículo esquerdo ou com eletrodo transvenoso posicionado em cardíaca esquerda (através do seio coronário), esta representando a configuração mais "clássica"19.

\section{Estimulação cardíaca temporária}

A estimulação cardíaca temporária é utilizada em casos de emergência ou de distúrbios reversíveis do ritmo cardíaco. Ela pode ser transcutânea, transvenosa ou epimiocárdica ${ }^{19}$.

\section{Estimulação transcutânea}

Essa técnica consiste na aplicação de estímulos elétricos diretamente na parede torácica pela aplicação de um par de eletrodos adesivos de grande superfície, colocados na parede torácica (anteroposterior, esternoápice ou laterolateral), que permitem, também, a desfibrilação e a monitorização eletrocardiográfica. Tem a vantagem de ser uma técnica não invasiva e de instalação praticamente imediata. A desvantagem é a necessidade do uso de alta energia, que habitualmente causa, 
também, estimulação muscular esquelética, principalmente dos músculos peitorais, que é tolerada por alguns pacientes e, em outros, exige sedação ${ }^{19}$.

\section{Estimulação transvenosa}

Nostranstornos reversíveis, como intoxicações medicamentosas, infarto agudo do miocárdio ou distúrbios hidroeletrolíticos, a melhor opção para a estimulação temporária é o uso de eletrodos bipolares transvenosos, ligados a um gerador de pulso externo. Esse tipo de estimulação traz como principal vantagem o fato de ser absolutamente indolor e por utilizar os dois polos de estimulação (cátodo e ânodo) no interior do coração ${ }^{19}$.

\section{Estimulação epimiocárdica}

Esse método tem sido empregado unicamente em pacientes submetidos à cirurgia cardíaca a céu aberto, nos quais os transtornos do ritmo cardíaco, permanentes ou reversíveis, ocorrem pelo manuseio das regiões vizinhas ao sistema de condução. Utilizam-se fios condutores de pequeno diâmetro que têm uma de suas extremidades implantada diretamente no miocárdio e a outra exteriorizada através da pele. Em caso de necessidade, são conectados ao gerador externo durante o período pós-operatório imediato e, posteriormente, removidos por tração ${ }^{19}$.

\section{Estimulação cardíaca artificial permanente}

Os dispositivos atuais têm a capacidade de estimular e detectar os batimentos cardíacos próprios do paciente, podendo ser construídos com a capacidade de comandar apenas uma cavidade cardíaca (átrios ou ventrículos, dependendo do distúrbio do ritmo), ou duas câmaras sequencialmente, no caso dos marcapassos atrioventriculares. A escolha do tipo de marcapasso leva em consideração um conjunto de fatores, como a idade, o estado geral do paciente, o estado funcional dos átrios e a função ventricular; para tanto, o modo de estimulação deve envolver a obediência aos princípios fisiológicos, bem como respeitar a individualidade de cada paciente, os recursos pessoais e materiais disponíveis no serviço ${ }^{19 ; 20}$.

\section{Implante de cardioversor-desfibrilador (CDI)}

Atualmente, o implante de cardiodesfibriladores automáticos é realizado de forma muito semelhante ao de MP cardíaco artificial transvenoso. A principal diferença entre esses dois tipos de procedimento diz respeito ao diâmetro do caboeletrodo ventricular, que, pela necessidade de conter os "coils" que o estímulo do choque percorre e são específicos para a desfibrilação ventricular e apresentam maior diâmetro do que os cabos-eletrodos de marca-passo ${ }^{19}$.

As indicações atuais para o CDI, que seguem, basicamente, as orientações revisadas do American Heart Association - AHA e do Consenso Brasileiro. Dividem-se em três classes, sendo que a primeira diz respeito aos sobreviventes de parada cardíaca por fibrilação ou taquicardias ventriculares consequentes às causas irreversíveis e não transitórias; taquicardias ventriculares sustentadas, espontâneas, mal toleradas, sem alternativa terapêutica eficaz, entre outros casos ${ }^{21}$.

A segunda classe aborda casos de fibrilação ventricular ou taquicardias ventriculares espontâneas, sustentadas, em candidatos a transplante cardíaco, com condições familiares ou hereditárias de alto risco para taquicardias ventriculares letais, tais como síndrome do QT longo, entre outros. E, por fim, a terceira classe, que engloba, entre vários casos, síncopes de origens indeterminadas em pacientes sem taquicardias espontâneas ou induzidas; taquicardia ventricular incessante, etc $^{19}$.

\section{INDICAÇÕES}

Algumas premissas que devem ser lembradas quando se fala em indicações para a estimulação cardíaca artificial: O marcapasso definitivo está indicado sempre que existir bradicardia sintomática e irreversível ou risco de bradicardia grave relacionada a causas não removíveis. Quando a causa é transitória ou removível deve-se tentar tratamento farmacológico ou marcapasso temporário. Os átrios devem ser estimulados e/ou detectados, sempre que possível, procurandose manter o sincronismo atrioventricular em repouso e durante o exercício; os ventrículos devem ser estimulados na presença de bloqueio atrioventricular, a estimulação ventricular isolada somente é aceitável quando os átrios não são aproveitáveis, como na fibrilação atrial crônica ou em situações especiais (crianças, idade avançada, dificuldade técnica, doenças consumptivas, etc) $)^{22}$.

O marcapasso temporário pode ser indicado para os casos de tratamento de patologias reversíveis, até que ocorra o restabelecimento do paciente, procedimentos diagnósticos e profilaxia. Os estudos atuais comprovam que os resultados da estimulação cardíaca são positivos a longo prazo, salientando que sua indicação deve ser feita com rigor. O marca-passo cardíaco definitivo é indicado quando o quadro do paciente for irreversível e sintomático ${ }^{16}$.

Uma vez implantado o marcapasso cardíaco definitivo, o paciente deverá ter conhecimento sobre possíveis interferências em seu funcionamento, uma vez que esse é assunto de extrema importância e abrangência. Isso ocorre devido à presença de um circuito de sensibilidade presente no interior do gerador, que, além de receber sinais originados por batimentos cardíacos, poderá sentir outras fontes elétricas, dependendo da qualidade e da intensidade da interferência ${ }^{9}$.

\section{Perfil Epidemiológico Atual}

O cuidado de portadores de marcapasso definitivo, chama a atenção dos profissionais quanto ao déficit de conhecimento sobre a doença, os sintomas, as manifestações de alteração da autoimagem, o sentimento de deterioração precoce do corpo, a insegurança e angústia pela eventual falha no aparelho, a 
preocupação com o tempo de duração das baterias, o medo de realizar atividades domésticas rotineiras, o desemprego, a alteração da função sexual e das atividades físicas, a perda do status social, familiar e profissional ${ }^{6,23}$.

O marca-passo cardíaco definitivo é indicado quando o quadro do paciente for irreversível e sintomático, sendo que a indicação mais comum para o implante de marcapasso definitivo é a doença do nó sinusal de acordo com as Diretrizes de 2015 da DECA/SBCCV ${ }^{16 ; 22}$.

Ao analisar o cenário epidemiológico atual, pode-se fazer a seguinte leitura:

Tabela 1. Internações por Unidade da Federação segundo Procedimento no período de 2009 a 2015.

\begin{tabular}{|c|c|c|c|}
\hline TIPOS DE MARCAPASSO & BRASIL & REGIÃO NORTE & RONDÔNIA \\
\hline Implante de marcapasso de câmara dupla transvenoso & 85.643 & 3.002 & 132 \\
\hline Implante de marcapasso de câmara única transvenoso & 23.796 & 764 & 79 \\
\hline Implante de marcapasso de câmara dupla epimiocárdico & 470 & 61 & 40 \\
\hline Implante de marcapasso de câmara única epimiocárdico & 848 & 79 & 29 \\
\hline Implante de marcapasso temporário transvenoso & 26.104 & 774 & 6 \\
\hline $\begin{array}{l}\text { Implante de marcapasso cardíaco multi-sítio endocavitário com reversão para } \\
\text { epimiocárdico por toracoto }\end{array}$ & 285 & 3 & 1 \\
\hline Implante de marcapasso cardíaco multi-sítio tranvenoso & 2.497 & 119 & 5 \\
\hline Total & 139.643 & 4.802 & 292 \\
\hline
\end{tabular}

Fonte: extraído DATASUS (BRASIL, 2017)24

De acordo com Lobo et al., (2010) a faixa etária média de idade dos pacientes submetidos a implantes de marcapasso em seu estudo realizado entre dezembro de 2005 a dezembro de 2006 foi de 73,72 anos (36-95 anos), com maior prevalência na faixa entre 70 e 90 anos. 6 Faz-se necessário o conhecimento a respeito do perfil dos pacientes uma vez que os estudos demonstram uma variabilidade nesses dados quando analisados por patologias específicas, como apontam ${ }^{25}$ pacientes chagásicos que apresentam menor faixa etária que pacientes não chagásicos; este estudo apontou que a média de idade do paciente chagásico foi de $(55,9 \pm 12,8$ anos) significativamente menor do que quando comparada ao não-chagásico $(68,32 \pm$ 14,9 anos).

Ao analisar os dados da tabela1, verificou-se que o marcapasso mais implantado no período corresponde ao de câmara dupla transvenoso totalizando cerca de $62 \%$ do total; este implante também é o mais seguro uma vez que apresenta a menor taxa de mortalidade $1,19 \%$, em relação ao marcapasso temporário transvenoso que apresenta a maior taxa de mortalidade com $21,13 \%^{24}$.

O perfil epidemiológico de determinada patologia e/ou procedimento é extremamente relevante, no sentido de nortear a elaboração de trabalhos que abordem temas relevantes que direcionem uma atuação profissional cada vez mais voltada para a excelência.

A capacidade do profissional em intervir na situação de assistência à saúde depende do que ele conhece dessa realidade e de sua competência técnico-cientifica. Essa competência pode ser avaliada de acordo com a qualidade do cuidado que oferece e do resultado de suas ações ${ }^{23}$.

\section{Papel da Enfermagem}

O planejamento da assistência de enfermagem como uma ação de gerência do cuidado ocorre por meio de um exercício contínuo de fazer escolhas e elaborar planos para realizar ou colocar uma determinada ação em prática. Ele envolve a avaliação das condições de saúde dos pacientes e, desse modo, o direcionamento das ações terapêuticas que serão empreendidas, bem como a delegação de atividades para equipe de enfermagem, organização dos diferentes procedimentos aos quais o paciente é submetido e previsão/ provisão dos materiais e recursos que são necessários. Para um planejamento eficaz, recomenda-se que o enfermeiro utilize indicadores, informações epidemiológicas e gerenciais para embasar suas ações e decisões ${ }^{26}$.

O Conselho Federal de Enfermagem (COFEN), normatiza, em âmbito nacional, a obrigatoriedade de haver enfermeiros em todas as unidades de serviços nos quais são desenvolvidas ações de enfermagem que envolva procedimentos de alta complexidade, comuns na assistência a pacientes críticos/ potencialmente $\operatorname{críticos}^{27}$.

A Sistematização da Assistência de Enfermagem (SAE) é um exemplo da prática de planejamento do enfermeiro, por meio da qual ele articula a dimensão assistencial e gerencial de seu trabalho. A ação de educar/capacitar a equipe de enfermagem é uma prática gerencial que assume características diferentes conforme as particularidades do contexto de atuação. Ao capacitar as equipes sob sua responsabilidade, o enfermeiro atua como facilitador da aquisição de saber, atualização profissional e capacidade de auto-organização, o que contribui para a realização de melhores práticas de cuidado ${ }^{26}$. 
Adicionalmente, incluem-se entre as responsabilidades privativas do enfermeiro: cuidados diretos de enfermagem a pacientes graves com risco de vida e cuidados de enfermagem de maior complexidade técnica e que exijam conhecimentos científicos adequados e capacidade de tomar decisões imediatas; e, como integrante da equipe de saúde, a participação nos programas e nas atividades de assistência integral à saúde individual e de grupos específicos, particularmente daqueles prioritários e de alto risco ${ }^{27}$.

As ações da Enfermagem na assistência com os pacientes portadores de marca-passo extremamente relevante. Elas incluem: orientar os pacientes portadores de marca-passo cardíaco definitivo sobre os principais cuidados que eles devem ter em seu domicílio e esclarecer suas principais dúvidas é de vital importância visto que, dessa forma, possíveis interferências e agravantes futuros poderão ser evitados, talvez desconhecidos pelos portadores desses instrumentos ${ }^{9}$.

Destarte, a formulação dos diagnósticos de enfermagem e do planejamento individualizado dos cuidados, guia o enfermeiro no desenvolvimento do raciocínio clínico para prever e prover os cuidados necessários a cada situação ${ }^{28}$.

Acredita-se que, desde o primeiro dia de internação, o enfermeiro exerce um papel fundamental na educação do paciente a ser submetido ao implante de marca-passo, uma vez que, no pré-operatório, ele é quem coleta os dados do paciente, identificando suas necessidades afetadas para poder planejar a assistência de enfermagem de maneira individualizada e sistematizada, incluindo a elaboração de um plano de alta ${ }^{9}$.

\section{Diagnósticos de Enfermagem para o Paciente Cardíaco Submetido a Implante de Marca-passo}

A avaliação criteriosa do enfermeiro por meio de instrumento de coleta de dados, utilizando-se da ciência da sistematização da Enfermagem, e do processo de Enfermagem, permite ao profissional fazer uma leitura dos riscos que o paciente possa vir a apresentar, bem como aqueles que o paciente já apresenta, servindo como base para as intervenções e os cuidados planejados de enfermagem no período pré, trans e pós de um procedimento de implante de marca-passo ${ }^{29}$.

De mesmo modo, a RESOLUÇÃO COFEN-358/2009 define o diagnóstico de Enfermagem como sendo o processo de interpretação e agrupamento dos dados coletados na primeira etapa, que culmina com a tomada de decisão sobre os conceitos diagnósticos de Enfermagem que representam, com mais exatidão, as respostas da pessoa, família ou coletividade humana em um dado momento do processo saúde e doença; e que constituem a base para a seleção das ações ou intervenções com as quais se objetiva alcançar os resultados esperados. ${ }^{28}$ Ainda quanto ao diagnóstico de Enfermagem a resolução $n=272$ de 27/08/2002/COFEN determina que ao enfermeiro incumbe privativamente: A implantação, planejamento, organização, execução e avaliação do processo de Enfermagem, que compreende as seguintes etapas: Consulta de Enfermagem, Histórico, Exame Físico, Diagnóstico de Enfermagem, Prescrição de Enfermagem e Evolução de Enfermagem, definindo, assim, que o enfermeiro após ter analisado os dados colhidos no histórico e exame físico, identificará os problemas de Enfermagem, as necessidades básicas afetadas e o grau de dependência, fazendo julgamento clínico sobre as respostas do indivíduo, da família e da comunidade, aos problemas e processos de vida vigentes ou potenciais ${ }^{29}$.

A fundamentação para a existência de um diagnóstico de Enfermagem é trazida pela Nanda Internacional destacando a existência e a atuação das diferentes áreas do conhecimento em saúde, dando ênfase ao lugar que a Enfermagem ocupa, assim: "Os enfermeiros lidam com respostas a condições de saúde/processos de vida entre indivíduos, famílias, grupos e comunidades. Essas respostas são a preocupação central dos cuidados de enfermagem..."26.

O diagnóstico de Enfermagem como uma linguagem própria e padronizada, conceituada como julgamento clínico das respostas do indivíduo, da família ou da comunidade aos processos vitais ou aos problemas de saúde, que fornecem a base para a seleção das intervenções de Enfermagem visam atingir resultados, pelos quais o enfermeiro seria o único responsável ${ }^{31}$.

Os diagnósticos de Enfermagem apresentam interpretações científicas de dados levantados, usados para orientar o planejamento, as implementações e a avaliação dos enfermeiros ${ }^{30}$.

Somente a partir da definição dos diagnósticos de enfermagem inferidos a partir do problema encontrado/apresentado pelo cliente, mediante o levantamento de dados alcançados por meio de coleta e posterior sistematização realizada por meio de análise do raciocínio clinico e pensamento crítico, poderá ser traçado um plano de intervenção e planejamento de cuidados. Portanto, cabe à Enfermagem identificar, durante a hospitalização, o diagnóstico do paciente e a intervenção relacionada visando melhorar seu estado físico, espiritual e social ${ }^{32}$.

Sendo assim, é de grande importância que o enfermeiro tenha conhecimento científico sobre a medicação e seus efeitos, a patologia de base do paciente e domine o conhecimento da linguagem padronizada por meio de uma taxonomia validada a fim de garantir uma assistência adequada e continuada por parte da equipe de Enfermagem, visando melhores resultados para o cliente.

Por meio deste estudo, foram selecionados e sugeridos alguns diagnósticos e intervenções que mais se aproximam do estado clínico dos pacientes submetidos a implantes de marca-passo, abordando todos os domínios e atentando ao paciente de maneira geral, sendo, assim, possível prestar uma assistência de qualidade. 
Tabela 2. diagnósticos de enfermagem e intervenções sugeridos a pacientes pré-trans-pós inserção de marca-passo

\begin{tabular}{|c|c|}
\hline Diagnóstico de enfermagem & Intervenções de enfermagem \\
\hline $\begin{array}{l}\text { Controle ineficaz da saúde relacionado ao conhecimento deficiente e } \\
\text { não aceitação do estado de saúde }\end{array}$ & $\begin{array}{l}\text { Promover educação em saúde; } \\
\text { Explicar os riscos da não adesão ao tratamento; } \\
\text { Conscientizar sobre a necessidade do regime terapêutico. }\end{array}$ \\
\hline $\begin{array}{l}\text { Insônia relacionada ao procedimento/mudança de ambiente/agentes } \\
\text { estressores e excesso de ruídos }\end{array}$ & Promover ambiente adequado minimizando estressores; \\
\hline Padrão do sono prejudicado, relacionado à ansiedade & $\begin{array}{l}\text { Investigar as causas de alteração do padrão de sono promovendo } \\
\text { conforto e condições adequadas; } \\
\text { Propiciar ambiente o mais familiar possível. }\end{array}$ \\
\hline $\begin{array}{l}\text { Débito cardíaco diminuído, relacionado ao volume de ejeção e } \\
\text { frequência cardíaca alterada }\end{array}$ & $\begin{array}{l}\text { Controle do equilíbrio hidroeletrolítico; } \\
\text { Administração de medicamentos; } \\
\text { Manutenção da hemodinâmica; } \\
\text { Monitorização multiparamétrica. }\end{array}$ \\
\hline $\begin{array}{l}\text { Intolerância à atividade, relacionada à incapacidade de realizar } \\
\text { pequenos esforços. }\end{array}$ & $\begin{array}{l}\text { Encorajar a realização de atividades simples; } \\
\text { Terapia de atividade; } \\
\text { Promoção de exercícios físicos simples. }\end{array}$ \\
\hline Déficit no autocuidado para banho, relacionado à imobilidade & $\begin{array}{l}\text { Assistência ao banho; } \\
\text { Apoio emocional; } \\
\text { Reforçar a necessidade de tentar higienizar-se sozinho. }\end{array}$ \\
\hline $\begin{array}{l}\text { Perfusão tissular periférica ineficaz, relacionado à incapacidade de } \\
\text { manter circulação adequada }\end{array}$ & $\begin{array}{l}\text { Administração de medicamentos; } \\
\text { Observar alterações nas características das extremidades; } \\
\text { Observar alterações na pele; } \\
\text { Monitorar oximetria de pulso }\end{array}$ \\
\hline $\begin{array}{l}\text { Conhecimento deficiente, relacionado a comportamento agitado/ } \\
\text { hostil/apático e ou relato de incompreensão da condição atual }\end{array}$ & $\begin{array}{l}\text { Educação quanto ao processo saúde/doença; } \\
\text { Aconselhamento. }\end{array}$ \\
\hline $\begin{array}{l}\text { Risco de síndrome do estresse por mudança, relacionado ao ambiente } \\
\text { nosocomial e mudança no padrão fisiológico }\end{array}$ & $\begin{array}{l}\text { Promover redução de mudança do ambiente; } \\
\text { Reduzir níveis de ansiedade; } \\
\text { Ambientar as mudanças; } \\
\text { Promover o enfrentamento; } \\
\text { Encorajar a trazer objetos pessoais. }\end{array}$ \\
\hline $\begin{array}{l}\text { Ansiedade, relacionado à mudança no estado de saúde (possibilidade } \\
\text { de cirurgia) e expectativa quanto aos resultados }\end{array}$ & $\begin{array}{l}\text { Explicar os processos do procedimento; } \\
\text { Ensinar técnicas de relaxamento. }\end{array}$ \\
\hline Medo, relacionado aos riscos do procedimento cirúrgico & $\begin{array}{l}\text { Oferecer apoio emocional tranquilizando quanto à realização do } \\
\text { procedimento. }\end{array}$ \\
\hline $\begin{array}{l}\text { Risco de infecção, relacionado ao local de inserção do cateter/Incisão } \\
\text { cirúrgica }\end{array}$ & $\begin{array}{l}\text { Observar a presença de sinais flogísticos; } \\
\text { Realizar curativo com técnica estéril a cada } 24 \text { horas. }\end{array}$ \\
\hline $\begin{array}{l}\text { Conforto prejudicado, relacionado à mudança de ambiente/restrição } \\
\text { de mobilidade }\end{array}$ & $\begin{array}{l}\text { Controlar fatores ambientais (temperatura, luminosidade, } \\
\text { privacidade...); } \\
\text { Reduzir ruídos. }\end{array}$ \\
\hline Dor aguda, relacionado à incisão cirúrgica & $\begin{array}{l}\text { Realizar avaliação da dor de forma ampla e sistemática (avaliar início, } \\
\text { frequência, amplitude, intensidade, etc.). }\end{array}$ \\
\hline
\end{tabular}

Fonte: Adaptado de NANDA Internacional 2015-201730.

\section{CONSIDERAÇÕES FINAIS}

Por meio deste estudo, foi possível compreender melhor a implantação dos dispositivos cardíacos implantáveis, sendo eles o MP e o CDI, seus tipos e indicações. Abordando a parte clínica voltada para os cuidados de enfermagem, profissionais que exercem papel fundamental nos pacientes submetidos a tal procedimento.

Sendo assim, o enfermeiro como profissional competente em realizar atividades que exijam conhecimento científico e técnico, deve estar capacitado para assumir os cuidados de tais procedimentos e mostrar-se extremamente importante em seu papel como profissional de saúde, a fim de evitar complicações e agravos à saúde dos pacientes submetidos à inserção de um estimulante cardíaco artificial, mais comumente chamado de marca-passo. 


\section{REFERÊNCIAS}

1. Hall JE. Tratado de fisiologia médica. 12. ed. Rio de Janeiro: Elsevier; 2011.

2. REY NA. Marcapasso cardíaco: indicações. Revista da Sociedade de Cardiologia do Rio Grande do Sul.2007 Set-Dez; 16(12): 1-5.

3. Borges JBC, Barros RT, Carvalho SMR, Silva MAM. Correlação entre a qualidade de vida, classe funcional e idade em portadores de marca-passo cardíaco. Rev. Bras Cir Cardiovasc. 2013 Jan-Mar; 28(1): 47-53. doi: http:// dx.doi.org/10.5935/1678-9741.20130008.

4. Ramos G, Ramos J Filho, Rassi A Jr, Pereira E, Gabriel S Neto, Chaves Enio. Marcapasso cardíaco artificial: considerações pré e per-operatórias. Rev. Bras. Anestesiol. 2003 Nov-Dec; 53(6): 854-862. doi: http://dx.doi.org/10.1590/ S0034-70942003000600015.

5. Gomes TB, Gomes LS, Antõnio IHF, Barroso TL, Cavalcante AMRZ, Sival MM, et al. Qualidade de vida pós-implante de marcapasso cardíaco artificial. Rev. Eletr. Enf. 2011 Out-Dez; 3(4): 735-42.

6. Lobo DEL, Gonçalves DP, Martins RS, Silva JLL. O perfil dos clientes do SUS submetidos a implante de marcapasso cardíaco definitivo em Hospital Universitário. Enferm. glob. 2010 Jun; (19): 1-8.

7. Mosquéra JAP, Pachóm Mateos JC, Vargas RNA, Costa ARB, Pachón Mateos JC. Aspectos epidemiológicos da estimulação cardíaca no Brasil - 11ㅇa ano do RBM - Registro Brasileiro de Marcapassos, desfibriladores e ressincronizadores cardíacos. Reblampa. 2006. 19(3):139-143.

8. Pachón JC, Mosquéra JAP, Pachon JC, Vargas RNA, Campo CM Neto, Costa AR. Aspectos epidemiológicos da estimulação cardíaca no Brasil - 12 a ano do RBM - Registro Brasileiro de Marcapassos, Desfibriladores e Ressincronizadores Cardíacos. Relampa. 2008.21(1): 5-12, 2008.

9. Aredes AF, Lucianeli JG, Dias MF, Bragada VCA, Dumbra APP, Pompeo DA Conhecimento dos pacientes a serem submetidos ao implante de marcapasso cardíaco definitivo sobre os principais cuidados domiciliares. Relampa. 2010, 23(1): 28-35

10. Silva RC, Ferreira MA. Um deslocamento do olhar sobre o conhecimento especializado em enfermagem: debate epistemológico. Rev Latino-am Enfermagem. 2008 Nov-Dez; 16(6): 1-7.

11. Martins C, Kobayashi RM, Ayoub AC, Leite MMJ. Perfil do enfermeiro e necessidades de desenvolvimento de Competência profissional. Texto contexto -enferm. 2006 Jul-Set; 15(3): 472-8. doi: http://dx.doi.org/10.1590/S010407072006000300012 .

12. Souza LP, Lima MG. Educação continuada em unidade de terapia intensiva: revisão da literatura. J Health BiolSci. 2015 Jan-Mar; 3(1): 39-45.

13. Mota WH, Kuster DE, Silva PCM, Mariano VT, Soares WKR, Souza LP. Diagnóstico de enfermagem ventilação Espontânea prejudicada, definição conceitual das características definidoras. Braz. J. Surg. Clin. Res. 2016 MarMaio; 14(3): 106-112.

14. Santos, JWN. Pires, DGH. Núñez, MAG. Riscos e complicações do uso de piercing oral: uma revisão sistemática. J Health Biol Sci. 2017 Jan-Fev; 5(1):95103.

15. Porth CM, Matfin G. Fisiopatologia. Rio de Janeiro: Guanabara Koogan; 2010.

16. Santos FCP, Rubio EM, Abreu LF, Barouche ER, Mateos JCP, Mateos EIP, et al. Marcapasso cardíaco: quando indicar e como usar. Rev. Fac. Ciênc. Méd. Sorocaba. 2008; 10(4): 5 - 7

\section{Como citar este artigo/How to cite this article:}

Mota WH, Saracini KC, Lima LCA, Algeri EDBO, Souza LP . Estimulação cardíaca artificial e suas implicações na enfermagem. J Health Biol Sci. 2018 Jan-Mar; 6(1):100-107.
17. Gontijo HT, Souza FSO, Avila V Neto, Gonçalves GA, Fazanaro GF, Sepulveda eletrodos de ventrículos esquerdo e direito. Relampa. 2014; 27(4):239-42.

18. Conti CR. Netter Ilustrações Médicas. 2. ed. Rio de Janeiro: Elsevier; 2015.

19. SCHETTINO; G. et al. Paciente crítico - diagnóstico e tratamento: hospital Sírio Libanês. 2. ed. Barueri/SP: Manole; 2012.

20. ANDRADE; J. C. S. et al. Diretrizes para implante Marcapasso cardíaco permanente. Arq Bras Cardiol. 2000 Maio; 74(5); 2000. doi: http://dx.doi. org/10.1590/S0066-782X2000000500009.

21. Oliveira DVR, Silva MF. Cardioversor-desfibrilador implantável: principais dúvidas dos pacientes no que se refere ao autocuidado após o implante. Relampa. 2010; 23(1):18-23. Eletrônicos Implantáveis (DCEI). Arq. Bras. Cardiol. 2007; 89(6): e210-e238.

23. Brasil VV. Qualidade de vida do portador de marcapasso cardíaco definitivo: antes e após o implante [tese]. São Paulo (SP): Universidade de São Paulo; 2001.

24. DATASUS. Sistema de Informações Hospitalares do SUS. MINISTÉRIO DA SAÚDE. 2017. http://tabnet.datasus.gov.br/cgi/tabcgi.exe?sih/cnv/qiuf.def.

25. Rincon LG, Rocha MOC, Baccarini PMT, Oliveira BG, Barros VCV, Barros MVL. Perfil clínico de pacientes chagásicos e não-chagásicos portadores de marcapasso cardíaco artificial. Rev. Soc. Bras. Med. Trop. 2006 Jun; 39(3): 245-249. doi: http://dx.doi.org/10.1590/S0037-86822006000300003.

26. Santos JLG, Pestana AL, Guerrero P, Meirelles BSH, Erdmann AL. Práticas de enfermeiros na gerência do cuidado em enfermagem e saúde: revisão integrativa. Rev. bras. enferm. 2013 Mar-Abr; 66(2): 257-263. doi: http://dx.doi. org/10.1590/S0034-71672013000200016.

27. Lino MM, Calil AM. O ensino de cuidados críticos/intensivos na formação do enfermeiro: momento para reflexão. Rer esc enferm. USP. 2008 Dez; 42(4): 777783. doi: http://dx.doi.org/10.1590/S0080-62342008000400022. outubro de 2009. Dispõe sobre a Sistematização da Assistência de Enfermagem e a implementação do Processo de Enfermagem em ambientes, públicos ou privados, em que ocorre o cuidado profissional de Enfermagem, e dá outras providências. Diário Oficial [da] República Federativa do Brasil. 2009 Out 23; Seção 1.

29. Brasil. Conselho Federal de Enfermagem. Resolução no 272, de 27 de agosto de 2002. Dispõe sobre a Sistematização da Assistência de Enfermagem - SAE nas Instituições de Saúde Brasileiras. Diário Oficial [da] República Federativa do Brasil. 2002 Ago 27; Seção 1.

30. North American Nursing Diagnosis Association. Diagnósticos de enfermagem da NANDA: definições e classificações 2015-2017. Porto Alegre: Artmed; 2015.

31. Pereira JMV, Cavalcanti ACD, Santanan RF, Cassiano KM, Queluci GC, Guimarães TCF. Diagnósticos de enfermagem de pacientes hospitalizados com doenças cardiovasculares. Esc Anna Nery. 2011 Dez; 15 (4):737-745. doi: http:// dx.doi.org/10.1590/S1414-81452011000400012.

32. Mariano S, Marques IR. Cardiomiopatia hipertrófica: atualização e assistência de enfermagem. Rev. bras. enferm. 2007; 60(5): 596-601. VM, et al. Análise comparativa do limiar agudo em unipolar e bipolar dos cabos-

22. Martinelli M Filho, editor. Diretrizes Brasileiras de Dispositivos Cardíacos

28. Brasil. Conselho Federal de Enfermagem. Resolução no 358, de 15 de 